\title{
Expression of Genes Related with Defense Responses against Pathogen Infections in Vitis flexuosa
}

\author{
Md Zaherul Islam ${ }^{1,3}$, Soon Young Ahn', Seon Ae Kim¹, Yong-Bum Kwack ${ }^{2}$, Hae Keun Yun ${ }^{1 *}$ \\ ${ }^{1}$ Department of Horticulture and Life Science, Yeungnam University, Gyeongsan 38541, Korea \\ ${ }^{2}$ Namhae Branch, National Institute of Horticultural and Herbal Science, Rural Development Administration, Namhae 52430, Korea \\ ${ }^{3}$ On-Farm Research Division, Bangladesh Agricultural Research Institute, Pabna 6600, Bangladesh
}

\begin{abstract}
Induced defense responses are regulated through a network of signal transduction pathways. Major signal transduction pathways in plants include salicylic acid (SA)- and jasmonic acid (JA)-mediated signaling pathways. In this study, we attempted to identify potential resistance mechanisms/pathways in Vitis flexuosa and the interaction between resistance and resistance related genes of $V$. flexuosa and several pathogens. To accomplish this, we investigated transcriptional expression of genes in the SA- and JA-mediated pathway and in the flavonoid biosynthesis pathway in V. flexuosa that had been infected by Elsinoë ampelina, Botrytis cinerea, Colletotrichum acutatum, Erysiphe necator, and Rhizobium vitis using real-time polymerase chain reaction (PCR) analysis. Quantitative real-time PCR revealed that R and R-related genes in $V$. flexuosa induced defense responses through expression of genes in SA-pathway rather than in the JA-pathway, and R-related genes were more closely correlated with lignin and phytoalexin biosynthesis than anthocyanin biosynthesis against different pathogen infections in $V$.flexuosa. Specifically, genes such as VfRLK586, VfRLK2422, VfRLK5099, VfRLK29610, VfRLK55257, VfRPS5-like4135, VfRPS5-like4832, VfRPS5-like20585, VfRPS5-like55532, $V f E D L 2, V f E D L 3, V f C X E 5585, V f C X E 12827$, and $V f C X E 13132$ showed close correlation with induction of genes in the SA-mediated pathway, lignin, phytoalexin, and anthocyanin biosynthesis. Genes including VfRLK2422, VfRLK5099, VfRLK29610, VfRPS5-like55532, $V f E D L 3$, and $V f C X E 12827$ were also correlated with the JA-mediated signaling pathway in induction of defense responses.
\end{abstract}

Keywords Disease resistance, Jasmonic acid, Real-time PCR, Salicylic acid, Vitis

\section{INTRODUCTION}

Plants have evolved complex recognition and response mechanisms to protect themselves against pathogens. Diseases occur when pathogens are able to avoid early detection by plant defense mechanisms (Feys and Parker 2000). One of the mechanisms of plant resistance to disease is conferred by resistance $(\mathrm{R})$ genes that generate products recognizing a pathogen's avirulence (Avr) proteins (Martin 1999). Following R gene mediated pathogen recognition, effector triggered immunity (ETI), which comprises a variety of defense responses, are activated in the pathogen-attacked plants. These include production of reactive oxygen species, accumulation of salicylic acid (SA), and increased expression of pathogenesis-related (PR) genes (Tsuda et al. 2008; Vlot et al. 2009). ETI also is frequently associated with the development of a hypersensitive response (HR) in which necrotic lesions form at the site(s) of pathogen entry. Subsequent to these responses in pathogen infected plants, ETI can trigger systemic SA accumulation and PR gene expression, as well as a long-lasting, broad-based resistance to subsequent pathogen infection known as systemic acquired resistance (Durrant and Dong 2004; Mishina and Zeier 2007; Vlot et al. 2009). Although, it is currently unclear how substantially the signaling pathway(s) leads to ETI, SA plays a critical role in activating ETI resistance (Mishina and Zeier 2007; Tsuda et al. 2008; Vlot et al. 2009). Jasmonic acid (JA) and ethylene have also been reported to play a critical role in regulation of signaling pathways (Kunkel and Brooks 2002). Moreover, these signaling molecules are important to the production of antimicrobial compounds such as

Received August 8, 2016; Revised August 12, 2016; Accepted August 13, 2016; Published August 31, 2016

*Corresponding author Hae Keun Yun, haekeun@ynu.ac.kr, Tel: +82-53-810-2942, Fax: +82-53-810-4659 
phytoalexin, anthocyanin and lignin (Lamb and Dixon 1997; Pieters and Van Loon 1999; Larronde et al. 2003; Belhadj et al. 2006).

Cultivated grapevines (Vitis spp.) are exposed to many pathogenic fungi, bacteria, and viruses (Wang et al. 2011). Because the application of commercial fungicides to control diseases is very expensive and causes various adverse effects on the environment, interest in the development of disease resistant grape cultivars is increasing. While cultivated grape species (Vitis vinifera L.) are highly susceptible to many diseases (Wang et al. 2011), wild cultivars such as $V$. riparia, $V$. rupestris, and $V$. rotundifolia

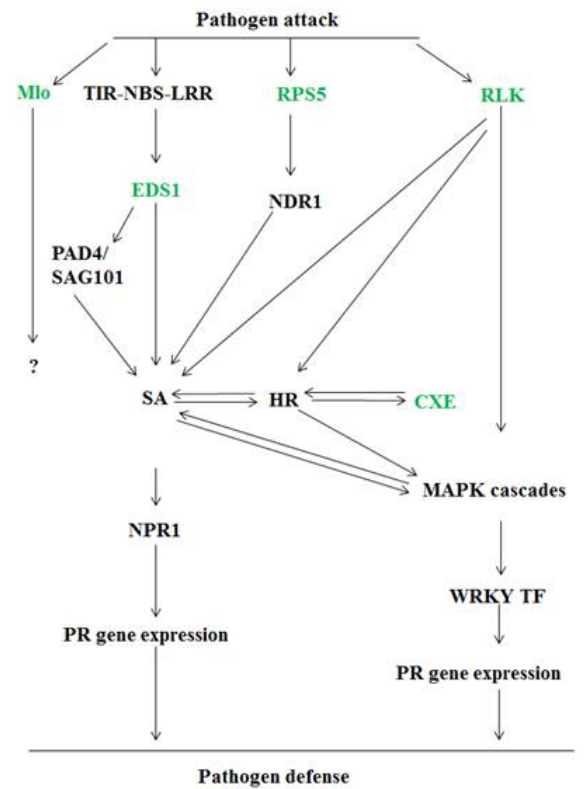

Fig. 1. Model depicting the interactions between pathogens and plant defense signaling cascade. Different $\mathrm{R}$ and $\mathrm{R}$ related genes of Vitis flexuosa are identified in this model.

CXE: carboxylesterse, EDS1: enhanced disease susceptibility 1, HR: hypersensitive response, MAPK: mitogen-activated protein kinases, Mlo: mildew resistance locus o, NDR: negative differentiation regulator, NPR1: non-expressor of pathogenesisrelated genes 1,PAD4/SAG101: phytoalexin deficient4/ senescence associated gene101, PR: pathogenesis related gene, RLK: receptor-like protein kinase, RPS5: resistance to pseudomonas syringae 5, SA: salicylic acid, TIR-NBS-LRR: toll interleukin-1 receptornucleotide binding site-leucine rich repeat, WRKY TF: WRKY transcription factor. are resistant to several important grapevine diseases (Eibach et al. 1989; Kortekamp et al. 2008; Reisch et al. 2012). Among Vitis species native to Korea, the wild cultivar $V$. flexuosa has been reported to show resistant responses such as expression of defense related genes against pathogen attacks (Ahn et al. 2012). We previously characterized 33 resistance and resistance related genes of five groups, receptor-like protein kinase $(R L K)$, resistance to Pseudomonas syringae 5-like (RPS5-like), mildew resistance locus o-like (Mlo-like), carboxylesterse ( $C X E$ ), and enhanced disease susceptibility1-like $(E D L)$ from transcriptome analysis based on the next generation sequencing of $V$. flexuosa inoculated with Elsinoë ampelina (Islam et al. 2015a; Islam et al. 2015b). R and R-related genes showed responses with different expression patterns against different pathogens in many plants (Fig. 1) (Baudouin et al. 1997; Ade et al. 2007; Afzal et al. 2008; Rietz et al. 2011). Therefore, this study was conducted to investigate the interaction between R and R-related genes of $V$. flexuosa and different pathogens and to identify potential resistance mechanisms/pathways in $V$. flexuosa by investigating transcriptional expression of genes in two major pathways, the SA and JA pathway, as well as in flavonoid biosynthesis pathway-related genes after infection with E. ampelina, Botrytis cinerea, Colletotrichum acutatum, Erysiphe necator, and Rhizobium vitis.

\section{MATERIALS AND METHODS}

\section{Plant materials and pathogens}

Leaves of $V$. flexuosa VISKO001 maintained in a grapevine germplasm collection field of Yeungnam University, Gyeongsan, Korea, were used for gene expression analysis following pathogen inoculation. The pathogens used in this study were virulent strains of E. ampelina (EA-1), B. cinerea (B1035), and C. acutatum (Co601), which were isolated from infected grapes by Dr. W.K. Kim, National Academy of Agricultural Science, RDA, Korea, E. necator collected from naturally infected leaves in the grape germplasm of Yeungnam University, Gyeongsan, and $R$. vitis strain Cheonan 493, which was kindly provided by Prof. J.S. Cha, Chungbuk National University, Korea. 


\section{Inoculation of pathogens}

Spore suspension $\left(10^{6}\right.$ spores $\left./ \mathrm{ml}\right)$ of E. ampelina, $C$. acutatum, and $E$. necator in distilled water and $B$. cinerea in $0.24 \%$ potato dextrose broth solution were sprayed for inoculation. Additionally, $20 \mu \mathrm{l}$ cell suspensions (OD $600=1)$ of $R$. vitis were dropped onto the wounded portions of leaves that had been injured slightly with a pencil tip. Leaves were then incubated in a moist box at $22^{\circ} \mathrm{C}$ to $28^{\circ} \mathrm{C}$ for 48 hours and harvested at the indicated time points $(0,1$, $6,12,24$, and 48 hours post inoculation [hpi]), immediately frozen in liquid nitrogen and then stored at $-80^{\circ} \mathrm{C}$ for future use.

Table 1. Primer sequences of genes used for real time PCR.

\begin{tabular}{|c|c|c|}
\hline Gene & Primers & Sequences $\left(5^{\prime}-3^{\prime}\right)$ \\
\hline \multirow[t]{2}{*}{ Phenylalanine ammonia-lyase $(P A L)$} & $\mathrm{FP}^{\mathrm{z})}$ & TGAACAATGGCGAAAGTGAGAA \\
\hline & $\mathrm{RP}^{\mathrm{z})}$ & TCTCTTGCGCTCTCAACCTCTT \\
\hline \multirow[t]{2}{*}{ Isochorismate synthase (ICS) } & FP & CGGTTTAGAGGACCACGATTTG \\
\hline & $\mathrm{RP}$ & GGACTTCCAATCGTCCAATGAG \\
\hline \multirow[t]{2}{*}{ Non-expressor of pathogenesis-related genes $1(N P R l)$} & FP & GACTTCAGTGGCAGTAGCAGCA \\
\hline & $\mathrm{RP}$ & AACCTCTGAGGAAAGGGATTCG \\
\hline \multirow[t]{2}{*}{ Pathogenesis related gene $1(P R l)$} & FP & GCCTACGCCCAGAATTATGCTA \\
\hline & $\mathrm{RP}$ & GCCCACCTGAATGCACTAGATT \\
\hline \multirow[t]{2}{*}{ Pathogenesis related gene $10(P R 10)$} & FP & ATGAGCTCAAAGTGGTGGCTTC \\
\hline & $\mathrm{RP}$ & CTTGGTGTGGTACTTGCTGGTG \\
\hline \multirow[t]{2}{*}{ Chitinase 1a (Chitla) } & FP & CAACAACCTGGACTGCAACAAC \\
\hline & $\mathrm{RP}$ & TCACATCACCATAGCAGAGTGGA \\
\hline \multirow[t]{2}{*}{ Lipoxygenase $(L O X)$} & FP & AACCTTGCGAGGTAATGGTCAA \\
\hline & $\mathrm{RP}$ & TACCACCAAGTACCGGTCGAGT \\
\hline \multirow[t]{2}{*}{ Allene oxide synthase $(A O S)$} & FP & GAGCTGTTCCTGCGTTACGATT \\
\hline & $\mathrm{RP}$ & GCTCAAAAACTGGCTCGCTTTA \\
\hline \multirow{2}{*}{ Allene oxide cyclase $(A O C)$} & FP & CTACACGGGAGACCTGGAGAAG \\
\hline & $\mathrm{RP}$ & СТСССТТСТTСССТGGAАСАТT \\
\hline \multirow[t]{2}{*}{ Plant defensing1.2 (PDF1.2) } & FP & GTGATGTTGGAAGCAAAGGTCT \\
\hline & $\mathrm{RP}$ & ATTTGGCATGACAAGCTCCAT \\
\hline \multirow[t]{2}{*}{ Chitinase 1b (Chit1b) } & FP & CGCCATCAAGTTCAGACACATC \\
\hline & $\mathrm{RP}$ & TGGAGCCTTTACCACATTCGAT \\
\hline \multirow{2}{*}{ 4-Coumarate-CoA ligase1 (4CL) } & FP & CGACAACCCCAACCTGTATTTC \\
\hline & $\mathrm{RP}$ & ATTGCTGCACCAACTCTCAATG \\
\hline \multirow[t]{2}{*}{ Chalcone synthase $(\mathrm{CHS})$} & FP & AGTTCAAGCGCATGTGTGAAAA \\
\hline & $\mathrm{RP}$ & CTTCAACCACCACCATGTCTTG \\
\hline \multirow[t]{2}{*}{ Stilbene synthase (STS) } & FP & CGAGCACATGTCTGAGTTGAAG \\
\hline & $\mathrm{RP}$ & GGTGATCTTTGATTTGGGTTGA \\
\hline \multirow[t]{2}{*}{ Cinnamyl alcohol dehydrogenase (CAD) } & FP & CACTGAGTCACTTTGGGCTGAA \\
\hline & $\mathrm{RP}$ & CCGATCAGAAGAGCTGATGACA \\
\hline \multirow[t]{2}{*}{ Chalcone isomerase $(\mathrm{CHI})$} & FP & TACACTGACGCAGAAGCCAAAG \\
\hline & $\mathrm{RP}$ & GACCCATCTTTGGAGAAGCTCA \\
\hline \multirow[t]{2}{*}{ Dihydroflavonol reductase $(D F R)$} & FP & TGTCTCCAAGACACTGGCTGAG \\
\hline & $\mathrm{RP}$ & TCGGGGAAAGAGCAGTTATGAG \\
\hline \multirow[t]{2}{*}{ Anthocyanidin synthase (ANS) } & FP & AAGATCATCCTGAAGCCACTGC \\
\hline & $\mathrm{RP}$ & TGGGTCTTCCTGAAGAGCTTGT \\
\hline \multirow[t]{2}{*}{ Beta-actin } & FP & ACGAGAAATCGTGAGGGATG \\
\hline & $\mathrm{RP}$ & ATTCTGCCTTTGCAATCCAC \\
\hline
\end{tabular}

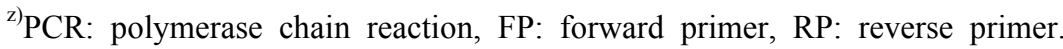




\section{RNA isolation and real-time polymerase chain reaction (PCR) analysis}

Total RNA was isolated from grapevine leaves using a slightly modified pine tree method (Chang et al. 1993). Total RNA concentration and quality were assessed using a Nano Drop spectrophotometer (ACTGENE ASP-3700; ACTGene, Inc., Piscataway, NJ, USA) and agarose gel electrophoresis. First-strand cDNA was synthesized from 500 ng total RNA using a GoScript ${ }^{\mathrm{TM}}$ Reverse Transcription System $\left(\right.$ GoScript $^{\mathrm{TM}}$ Reverse Transcription System; Promega, Madison, WI, USA), then used as a template for PCR. Real-time PCR with first-strand cDNA from RNA was performed using SYBR Premix Ex (SYBR Premix Ex Taq; TaKaRa Bio Inc., Osaka, Japan) as the

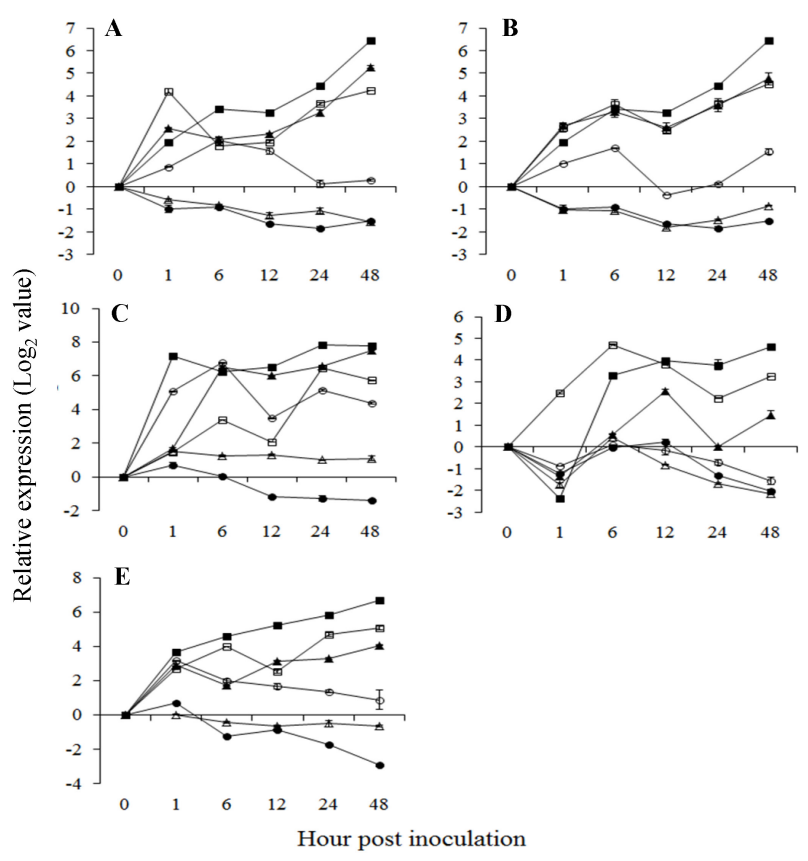

Fig. 2. Expression pattern of salicylic acid pathway related genes in Vitis flexuosa against Elsinoë ampelina (A), Botrytis cinerea (B), Colletotrichum acutatum (C), Erysiphe necator (D), and Rhizobium vitis (E). Phenylalanine ammonia-lyase $(P A L,-\odot)$, isochorismate synthase (ICS, -), non-expressor of pathogenesis-related genes $1(N P R 1, \triangle)$, pathogenesis related gene $1(P R 1, \mathbf{A})$, chitinase 1a (Chitla, $\square)$, pathogenesis related gene $10(P R 10,-)$. The error bars represent the standard error of the means of three independent replicates. fluorescent dye and a $\mathrm{C} 1000^{\mathrm{TM}}$ Thermal Cycler $\left(\mathrm{CFX} 96^{\mathrm{TM}}\right.$ Real-Time System; BioRad, Hercules, CA, USA). PCR amplification was conducted by subjecting the samples to $95^{\circ} \mathrm{C}$ for 30 seconds, followed by 40 cycles of $95^{\circ} \mathrm{C}$ for 5 seconds and $60^{\circ} \mathrm{C}$ for 30 seconds, and then a melting-curve program at $60^{\circ} \mathrm{C}$ to $95^{\circ} \mathrm{C}$ for 5 seconds. The relative transcript levels were calculated for treated samples relative to the non-treated samples according to the standard-curve method. All samples were analyzed in triplicate to ensure consistency of the results (Table 1).

\section{RESULTS}

To gain insight into defense signaling responses in $V$. flexuosa against attacks of pathogens, the expression of different $\mathrm{R}$ and $\mathrm{R}$ related genes, two major pathways (SA

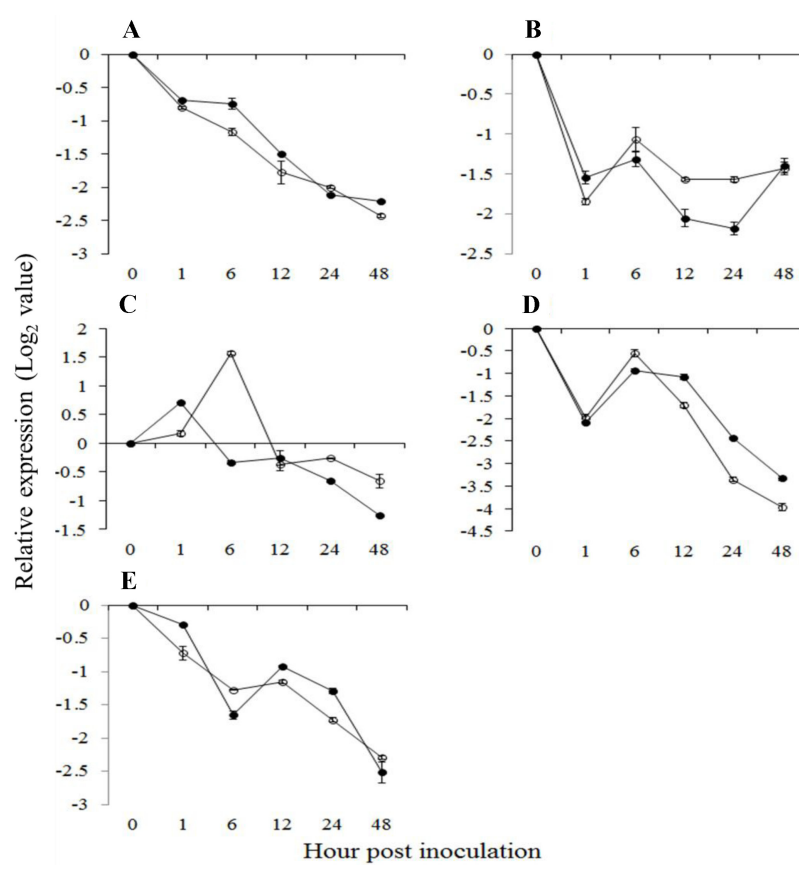

Fig. 3. Expression pattern of EDS1 interacting genes and upstream of salicylic acid pathway related genes in Vitis flexuosa against Elsinoë ampelina (A), Botrytis cinerea (B), Colletotrichum acutatum (C), Erysiphe necator (D), and Rhizobium vitis (E). Phytoalexin Deficient4 (PAD4, $\odot)$, Senescence Associated Gene101 (SAG101, -). The error bars represent the standard error of the means of three independent replicates. 
and JA-mediated pathway) and one secondary metabolite, flavonoid biosynthesis pathway-related genes were investigated after infection by five pathogens. Among two SA biosynthesis related genes, phenylalanine ammonia-lyase $(P A L)$ showed upregulated and isochorismate synthase (ICS) showed downregulated expression against $E$. ampelina (Fig. 2A), suggesting that SA biosynthesis was positively related to resistant responses to $E$. ampelina infection through the $P A L$ induction pathway. The signal transduction gene, non-expressor of pathogenesis-related genes $1(N P R l)$, showed downregulated expression in response to E. ampelina infections. Nuclear-localization of $N P R 1$ is required in SA-dependent expression of defense genes, including pathogenesis related gene 1 (PR1). Our results also showed upregulated expression of $P R 1$, pathogenesis related gene 10 (PR10), and chitinase 1a (Chit1a) against E. ampelina infection (Fig. 2A). In $C$.

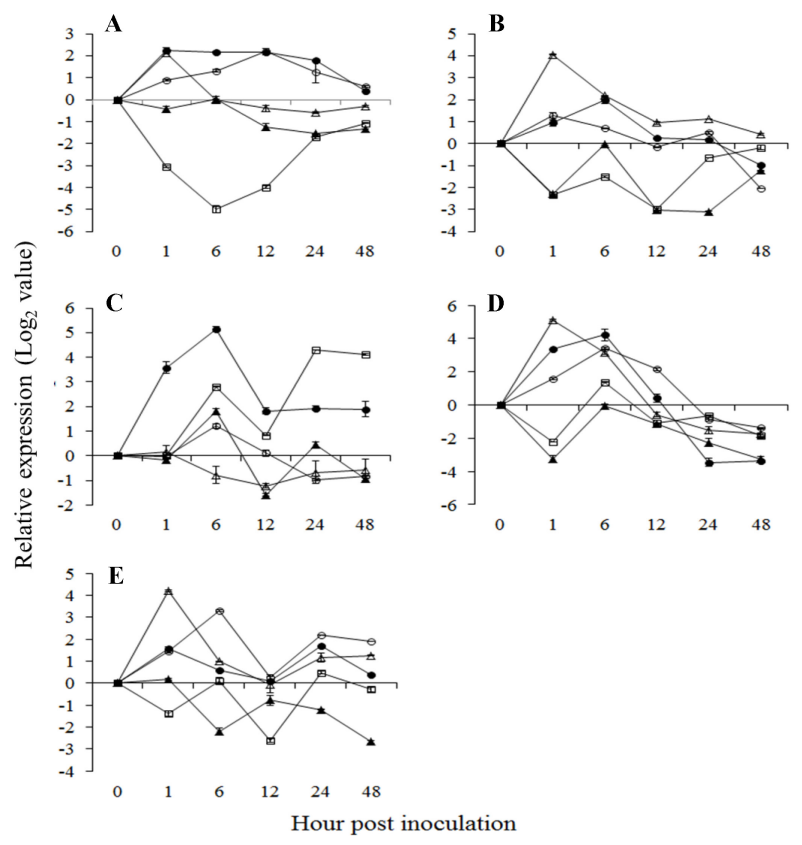

Fig. 4. Expression pattern of jasmonic acid pathway related genes in Vitis flexuosa against Elsinoë ampelina (A), Botrytis cinerea (B), Colletotrichum acutatum (C), Erysiphe necator (D), and Rhizobium vitis (E). Lipoxygenase $(L O X, \ominus)$, allene oxide synthase $(A O S,-)$, allene oxide cyclase $(A O C, \triangle)$, plant defensing1.2 (PDF1.2, $\mathbf{A})$, chitinase 1b (Chitlb, $\square)$. The error bars represent the standard error of the means of three independent replicates. acutatum inoculated-leaves, the expression of PR10 increased at 24 hours after inoculation, declined at 48 hours (Fig. 2C). The expression of two EDS1 interacting genes upstream of SA synthesis, Phytoalexin Deficient4 (PAD4) and Senescence Associated Gene101 (SAG101) were shown to be down-regulated in response to all pathogen infections (Fig. 3). In this study, four major JA-mediated defense-related genes, lipoxygenase $(L O X)$, allene oxide synthase $(A O S)$, allene oxide cyclase $(A O C)$, plant defensing1.2 (PDF1.2), and chitinase1b (Chit1b), were tested. PDF1.2, the JA pathway related defense marker gene, was not upregulated and Chit $1 b$ was downregulated in response to E. ampelina (Fig. 4A). Among the three JA biosynthesis related genes, $L O X$ and $A O S$ were shown to be upregulated at all time points, and $A O C$ was upregulated at

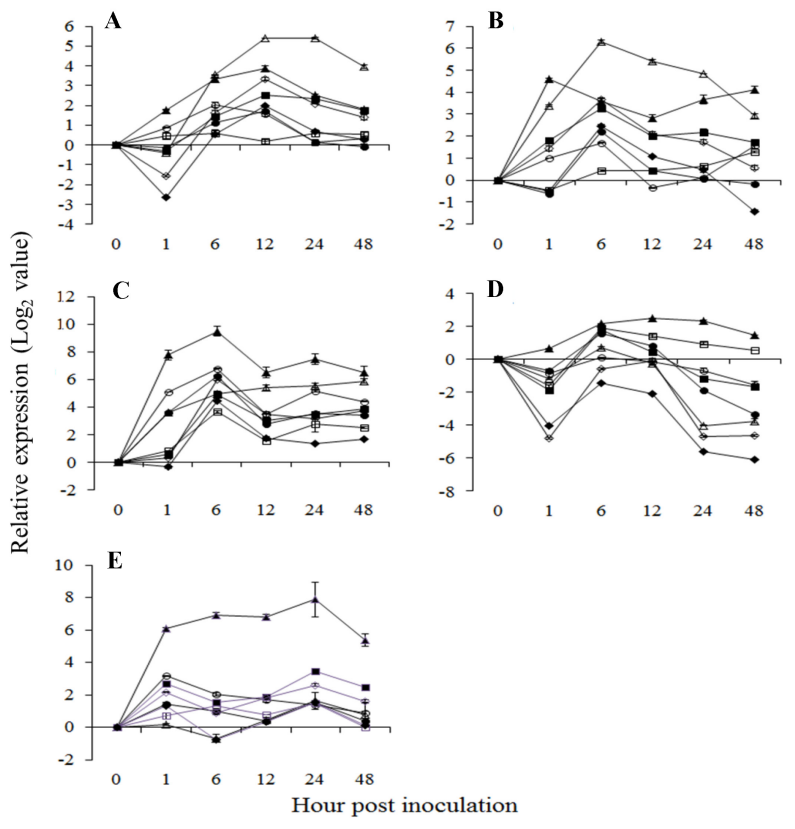

Fig. 5. Expression patterns of flavonoid biosynthesis pathway related genes in Vitis flexuosa against Elsinö ampelina (A), Botrytis cinerea (B), Colletotrichum acutatum (C), Erysiphe necator (D), and Rhizobium vitis $(\mathrm{E})$. Phenylalanine ammonia-lyase $(P A L, \Theta)$, 4-Coumarate-CoA ligase1 (4CL, - -), chalcone synthase $(C H S, \triangle)$, stilbene synthase $(S T S, \mathbf{A})$, cinnamyl alcohol dehydrogenase $(C A D, \square)$, chalcone isomerase ( $\mathrm{CHI}$, -), dihydroflavonol reductase $(D F R, \diamond)$, anthocyanidin synthase $(A N S, \bullet)$. The error bars represent the standard error of the means of three independent replicates. 


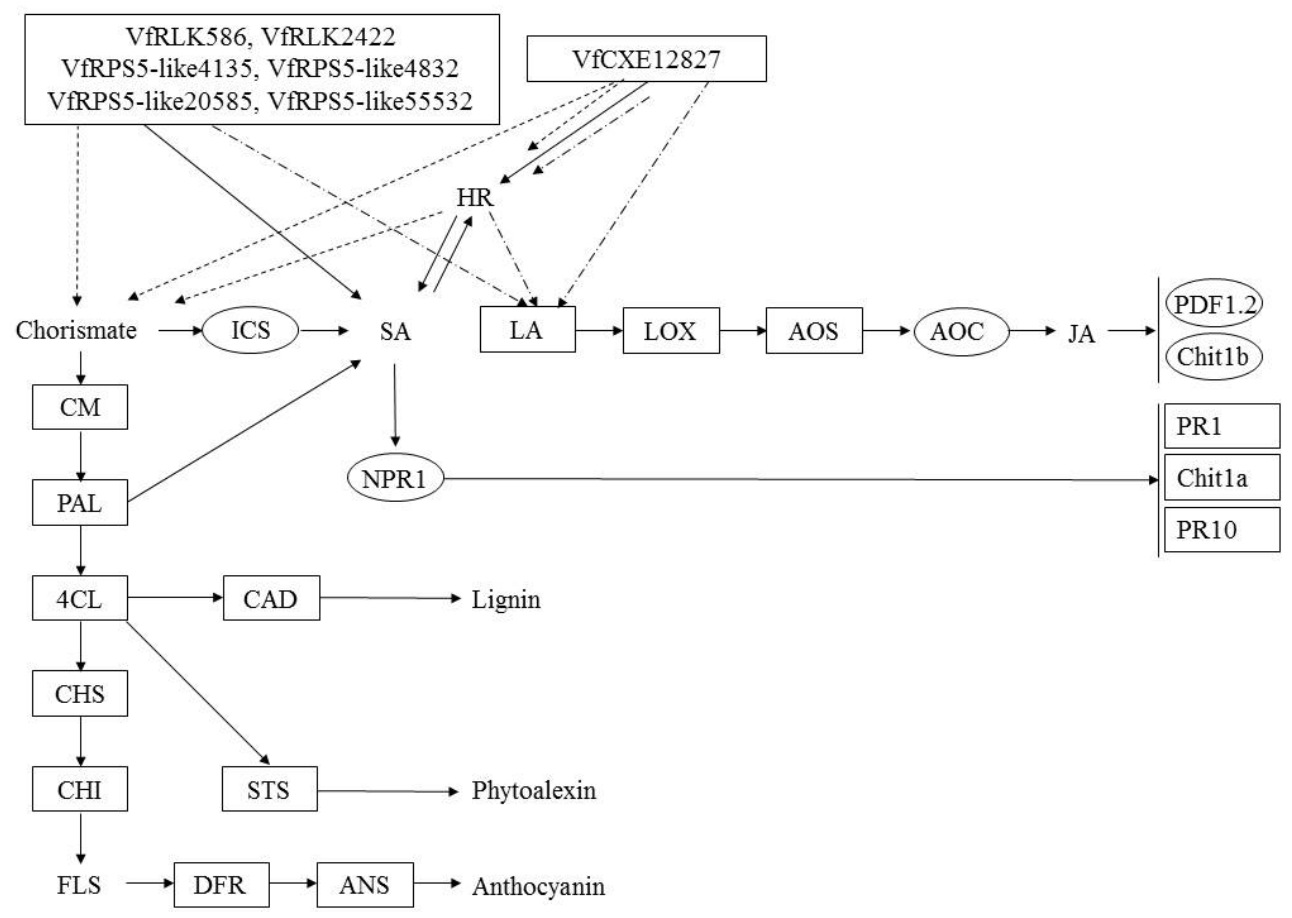

Fig. 6. A diagram of interaction between different $\mathrm{R}$ and $\mathrm{R}$ related genes of Vitis flexuosa and Elsinoë ampelina. In this diagram known defense signaling pathways-SA and JA and flavonoid biosynthesis related major genes are used. Upregulated genes are shown by rectangular boxes.

HR: hypersensitive response, ICS: isochorismate synthase, SA: salicylic acid, LA: linoleic acid, LOX: lipoxygenase, $A O S$ : allene oxide synthase, $A O C$ : allene oxide cyclase, JA: jasmonic acid, PDF1.2: plant defensing1.2, Chit1b: chitinase 1b, CM: chorismate mutase, PAL: phenylalanine ammonia-lyase, NPR1: non-expressor of pathogenesisrelated genes 1, PR1: pathogenesis related gene 1, Chit1a: chitinase 1a, PR10: pathogenesis related gene 10, 4CL: 4-Coumarate-CoA ligase1, $C A D$ : cinnamyl alcohol dehydrogenase, $C H S$ : chalcone synthase, $C H I$ : chalcone isomerase, STS: stilbene synthase, FLS: flavonol synthase, DFR: dihydroflavonol reductase, ANS: anthocyanidin synthase.

1 hpi against E. ampelina (Fig. 4A). The flavonoid biosynthesis related genes, $P A L$, 4-coumarate: CoA ligase (4CL), chalcone synthase (CHS), stilbene synthase (STS), cinnamyl alcohol dehydrogenase $(C A D)$, chalcone isomerase $(C H I)$, dihydroflavonol-4-reductase $(D F R)$, and anthocyanidin synthase $(A N S)$ were all upregulated in response to E. ampelina infection (Fig. 5A), suggesting that $V$. flexuosa protects itself by lignification and production of phytoalexin and anthocyanin against $E$. ampelina. $\mathrm{R}$ and $\mathrm{R}$ related genes in $V$. flexuosa such as VfRLK586, VfRLK2422, VfRPS5-like4135, VfRPS5-like4832, VfRPS5-like20585, VfRPS5-like55532, and VfCXE12827, showed upregulated expression during E. ampelina infection (Fig. 6). Therefore, VfRLK, VfRPS5-like, and $V f C X E$ genes may have strong involvement in the SA and
HR signaling pathway and strong correlation with lignin, phytoalexin and anthocyanin biosynthesis during infection of E. ampelina. In the case of B. cinerea infection, SA, JA and flavonoid biosynthesis related genes showed the same expression pattern, except for $A O C$, which was upregulated at all time points (Fig. 2B, 4B, 5B). The VfRLK2422, VfRPS5-like20585, VfRPS5-like55532, VfEDL3, VfCXE5585, $V f C X E 12827$, and VfCXE13132 R-related genes were upregulated against $B$. cinerea infection (Fig. 7). These results indicate that the SA-mediated pathway, lignin, phytoalexin and anthocyanin biosynthesis pathway are also involved in grapevine defense against $B$. cinerea infection. In the case of $C$. acutatum infection, all genes in the SA-mediated pathway except ICS were upregulated (Fig. 2C), Chit1b (JA-mediated pathway related defense 


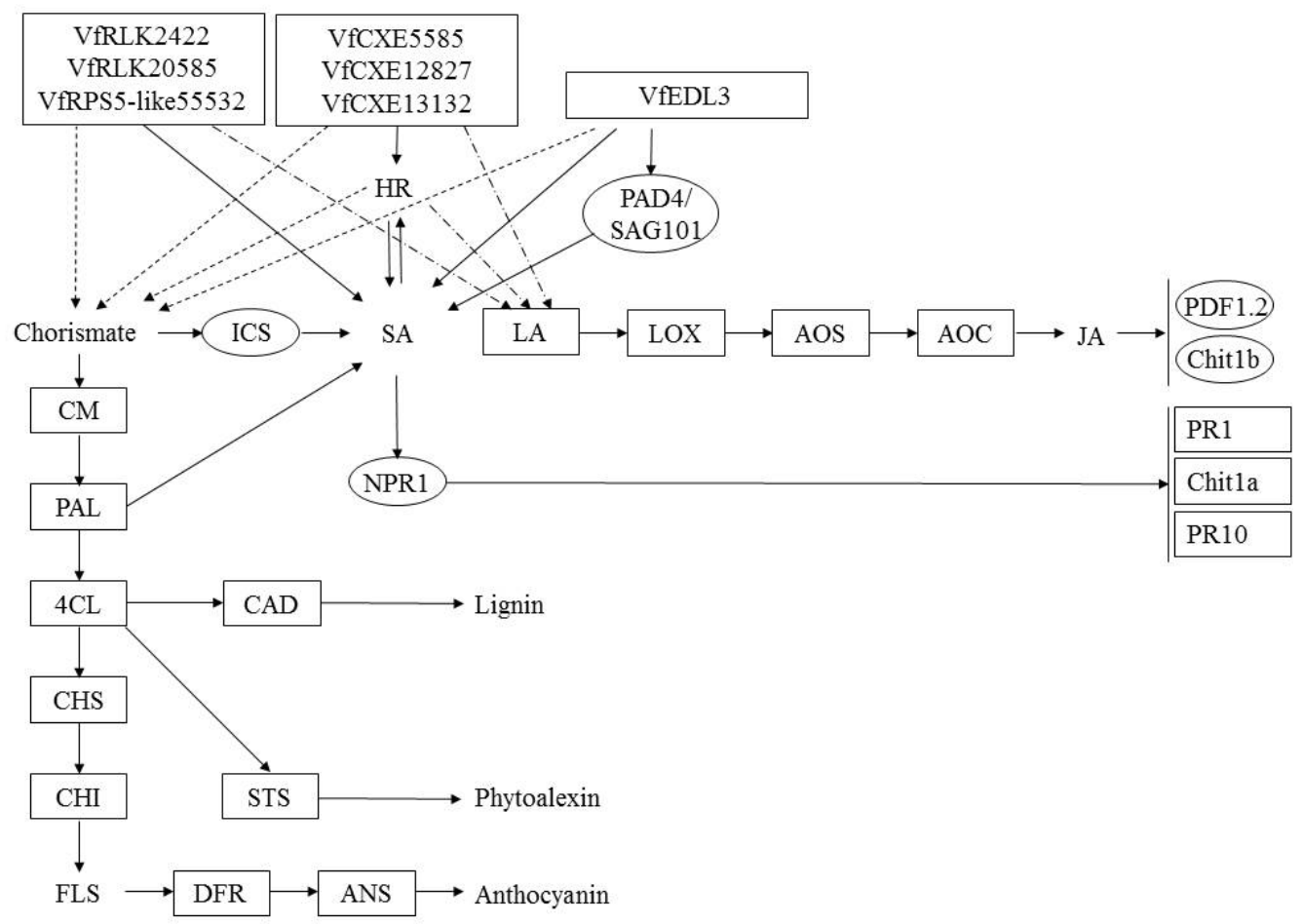

Fig. 7. A diagram of interaction between different $\mathrm{R}$ and $\mathrm{R}$ related genes of Vitis flexuosa and Botrytis cinerea. In this diagram known defense signaling pathways-SA and JA and flavonoid biosynthesis related major genes are used. Upregulated genes are shown by rectangular boxes.

HR: hypersensitive response, PAD4: phytoalexin Deficient4, SAG101: senescence Associated Gene101, ICS: isochorismate synthase, SA: salicylic acid, LA: linoleic acid, $L O X$ : lipoxygenase, $A O S$ : allene oxide synthase, $A O C$ : allene oxide cyclase, JA: jasmonic acid, PDF1.2: plant defensing1.2, Chit1b: chitinase 1b, CM: chorismate mutase, $P A L$ : phenylalanine ammonia-lyase, NPR1: non-expressor of pathogenesis-related genes 1, PR1: pathogenesis related gene 1, Chit1a: chitinase 1a, PR10: pathogenesis related gene 10, 4CL: 4-Coumarate-CoA ligase1, CAD: cinnamyl alcohol dehydrogenase, $C H S$ : chalcone synthase, CHI: chalcone isomerase, STS: stilbene synthase, FLS: flavonol synthase, DFR: dihydroflavonol reductase, ANS: anthocyanidin synthase.

gene) and $A O S$ (JA biosynthesis gene) were upregulated at all time points, and $P D F 1.2$ and $L O X$ were shown to be upregulated at some time points (Fig. 4C). Genes related to flavonoid biosynthesis also showed upregulated expression (Fig. 5C). VfRLK2422, VfRLK29610, VfRPS5-like55532, VfEDL3, VfCXE5585, VfCXE12827, and VfCXE13132 were shown to be upregulated during $C$. acutatum infection (Fig. 8). Therefore, VfRLK, VfRPS5-like, VfEDL, and $V f C X E$ genes may be closely involved in the SA and HR signaling pathway and may have close correlation with lignin, phytoalexin and anthocyanin biosynthesis during infection by C. acutatum. Moreover, the JA pathway may be involved in defense responses against $C$. acutatum infection in $V$. flexuosa. The SA-mediated pathway related defense genes PR1, PR10, and Chit1a were upregulated at all time points, whereas SA biosynthesis related genes were not upregulated in response to E. necator infection (Fig. 2D). Conversely, LOX, AOS, and AOC (JA biosynthesis genes) and Chitlb (JA-mediated pathway defense gene) were upregulated at 6 hpi after infection by E. necator (Fig. 4D). These results suggest that the JA-mediated pathway may be more involved than the SA-mediated pathway in the response of $V$. flexuosa against E. necator. Among flavonoid biosynthesis related genes, all of them were down regulated except lignin and phytoalexin biosynthesis related genes CAD and STS (Fig. 5D). VfRLK5099, VfRLK29610, VfRPS5-like4135, VfRPS5-like20585, and $V F C X E 12827$ were upregulated during $E$. necator infection 


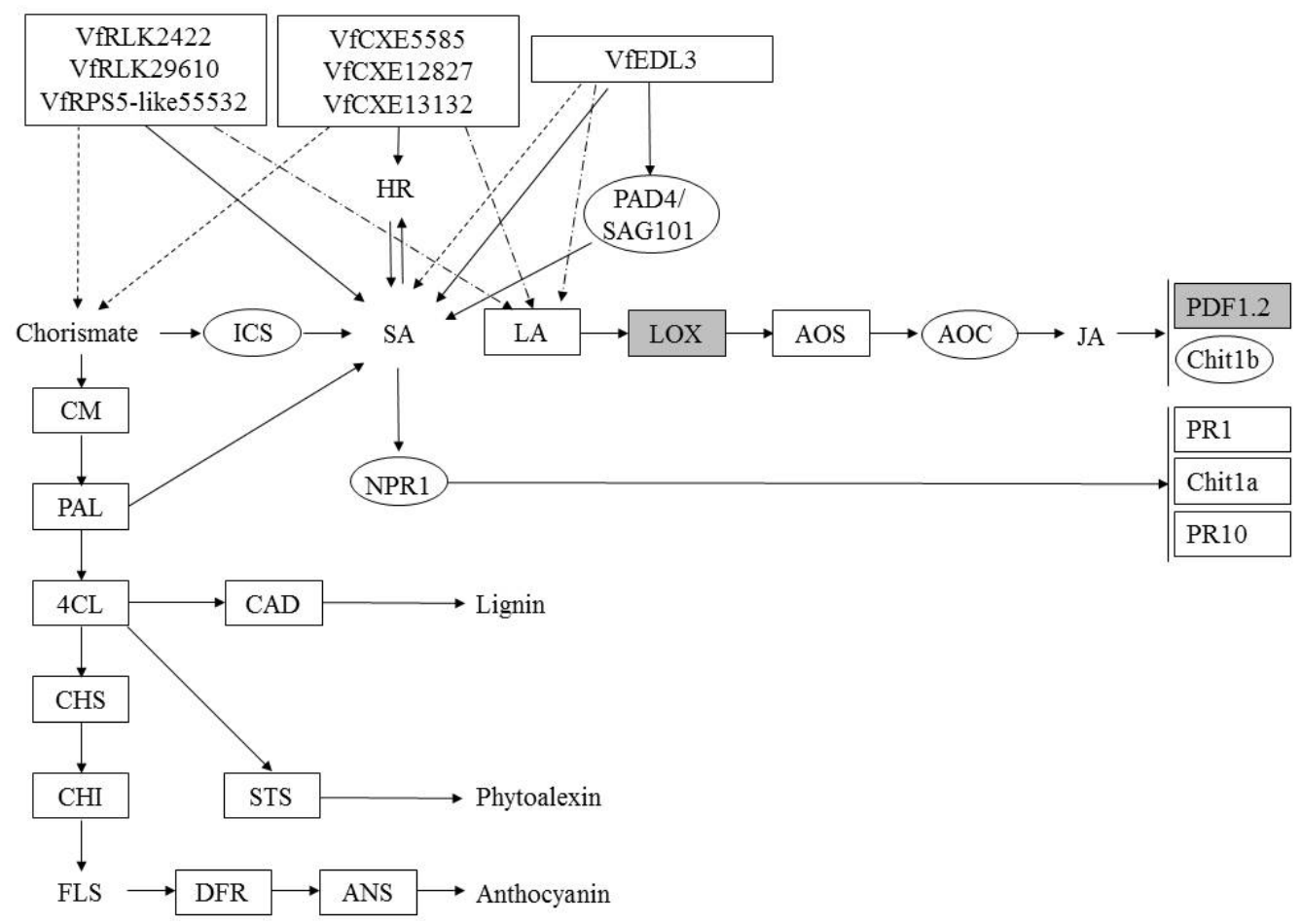

Fig. 8. A diagram of interaction between different $\mathrm{R}$ and $\mathrm{R}$ related genes of Vitis flexuosa and Colletotrichum acutatum. In this diagram known defense signaling pathways-SA and JA and flavonoid biosynthesis related major genes are used. Upregulated genes are shown by rectangular boxes. Some points upregulated genes are shown by rectangular shaded boxes.

HR: hypersensitive response, PAD4: phytoalexin Deficient4, SAG101: senescence Associated Gene101, ICS: isochorismate synthase, SA: salicylic acid, LA: linoleic acid, $L O X$ : lipoxygenase, $A O S$ : allene oxide synthase, $A O C$ : allene oxide cyclase, JA: jasmonic acid, $P D F 1.2$ : plant defensing1.2, Chit1b: chitinase 1b, CM: chorismate mutase, $P A L$ : phenylalanine ammonia-lyase, NPR1: non-expressor of pathogenesis-related genes 1, $P R 1$ : pathogenesis related gene 1, Chit1a: chitinase 1a, PR10: pathogenesis related gene 10, 4CL: 4-Coumarate-CoA ligase1, CAD: cinnamyl alcohol dehydrogenase, $C H S$ : chalcone synthase, $C H I$ : chalcone isomerase, STS: stilbene synthase, FLS: flavonol synthase, DFR: dihydroflavonol reductase, ANS: anthocyanidin synthase.

(Fig. 9). Therefore, VfRLK5099, VfRLK29610, VfRPS5like4135, VfRPS5-like20585, and VfCXE12827 may be correlated with the JA-mediated pathway in $V$. flexuosa against the powdery mildew pathogen, E. necator. In response to $R$. vitis infection, all tested genes related to the SA-mediated pathway except ICS and NPRI were upregulated (Fig. 2E), whereas the JA pathway related defense genes $P D F 1.2$ and Chit $1 b$ were downregulated, but the JA biosynthesis genes $L O X, A O S$, and $A O C$ were upregulated (Fig. 4E). All tested flavonoid biosynthesis related genes showed upregulation against $R$. vitis (Fig. 5E). VfRLK586, VfRLK2422, VfRLK5099, VfRPS5-like55532, VfEDL2, VfEDL3, VfCXE5585 and VfCXE13132 were upregulated in response to $R$. vitis infection (Fig. 10).

\section{DISCUSSION}

In our previous studies, 33 resistance and resistance related genes of five groups, RLK, RPS5-like, Mlo-like, $C X E$, and $E D L$, from $V$. flexuosa inoculated with $E$. ampelina were characterized and found to have different expression levels (Islam et al. 2015a; Islam et al. 2015b). These findings indicate that all of these genes may be involved in plant resistance responses against different pathogens in $V$. flexuosa native to Korea.

$R L K$ plays fundamental signaling roles in many different processes, including regulation of growth, development and disease resistance (Zhou et al. 1995; Shiu and Bleecker 2001; Swiderski and Innes 2001; Chen et al. 2003; Chen et 


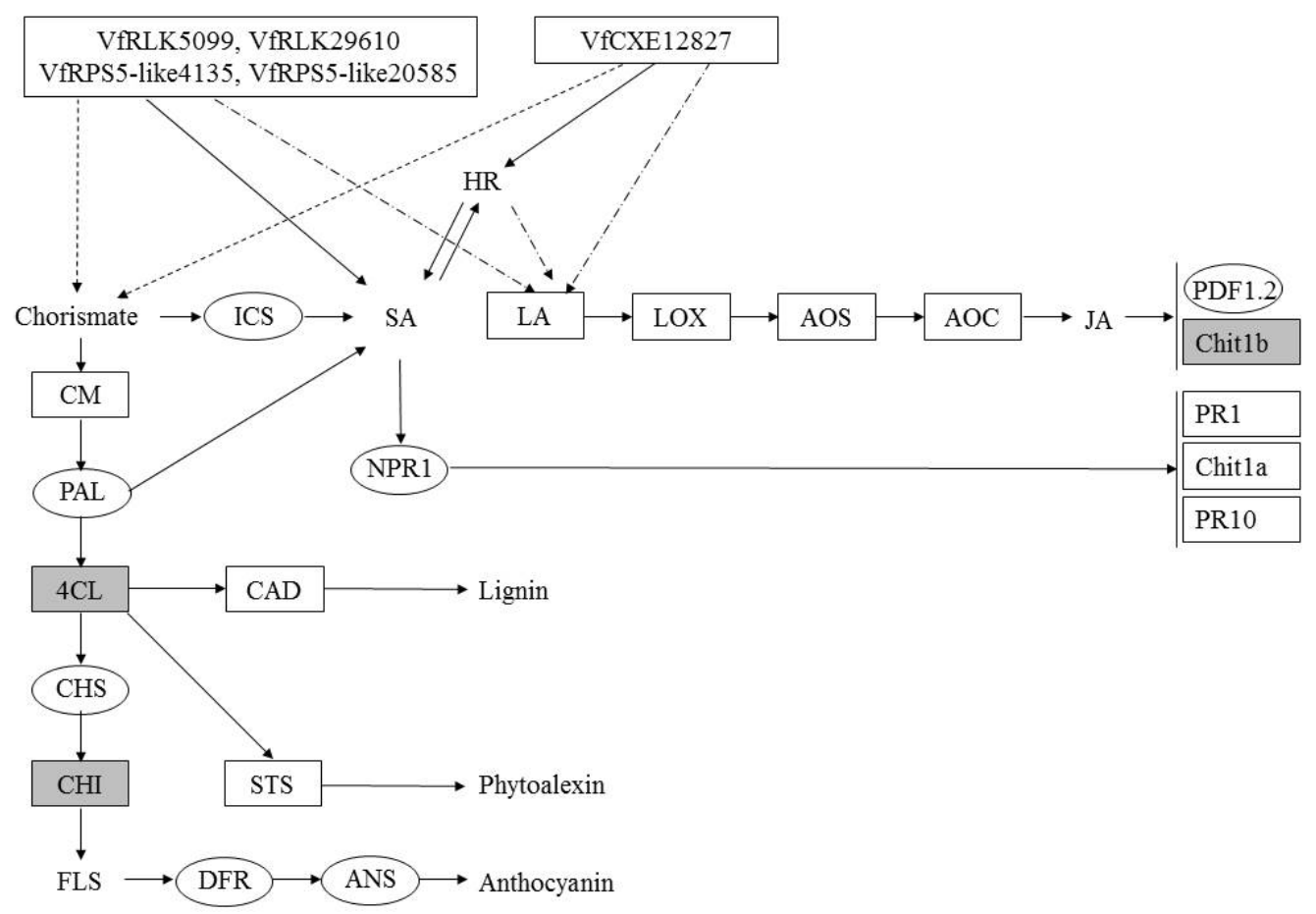

Fig. 9. A diagram of interaction between different $\mathrm{R}$ and $\mathrm{R}$ related genes of Vitis flexuosa and Erysiphe necator. In this diagram known defense signaling pathways-SA and JA and flavonoid biosynthesis related major genes are used. Upregulated genes are shown by rectangular boxes. Some points upregulated genes are shown by rectangular shaded boxes.

HR: hypersensitive response, PAD4: phytoalexin Deficient4, SAG101: senescence Associated Gene101, ICS: isochorismate synthase, SA: salicylic acid, LA: linoleic acid, $L O X$ : lipoxygenase, AOS: allene oxide synthase, $A O C$ : allene oxide cyclase, JA: jasmonic acid, PDF1.2: plant defensing1.2, Chit1b: chitinase 1b, CM: chorismate mutase, $P A L$ : phenylalanine ammonia-lyase, NPR1: non-expressor of pathogenesis-related genes 1, PRl: pathogenesis related gene 1, Chit1a: chitinase 1a, PR10: pathogenesis related gene 10, 4CL: 4-Coumarate-CoA ligase1, CAD: cinnamyl alcohol dehydrogenase, CHS: chalcone synthase, CHI: chalcone isomerase, STS: stilbene synthase, FLS: flavonol synthase, DFR: dihydroflavonol reductase, ANS: anthocyanidin synthase.

al. 2004; Afzal et al. 2008; Xu and Zhang 2015). The Arabidopsis RPS5 gene is activated by AvrPphB mediated cleavage of the protein kinase PBS1 and engages in downstream signaling molecules that activate the defense response (Feys and Parker 2000; Ade et al. 2007; Qi et al. 2012). Several CXE genes, including hsr203J (tobacco), Lehsr203 (tomato), BIG8.1 (Botrytis-induced grapevine), PepEST (Pepper), and SOBER1 (Arabidopsis), were characterized as hypersensitivity-related genes (Baudouin et al. 1997; Tronchet et al. 2001; Bézier et al. 2002; Ko et al. 2005). EDS1 plays an important role in the $\mathrm{R}$ gene mediated defense signaling pathway (Aarts et al. 1998; Rietz et al. 2011).

In cases of pathogen infection in plants, SA accumu- lation led to altered cellular redox status, partially reduced NPR1 and facilitated its localization to the nucleus (Dempsey et al. 2011). Moreover, NPRl and in the nucleus interacted with specific transcription factors to regulate gene expression (Dong 2004; Pieterse and Van Loon 2004). NPR1 has also been shown to negatively regulate SA accumulation by suppressing ICS1 expression, and NPR1-mediated downregulation of SA has been successfully activated by continuous escalation of SA levels in resistance responses (Dempsey et al. 2011). The defense response related genes $P R 1, P R 10$, and Chit1a are typical upregulated marker genes in the SA pathway (Chong et al. 2008). In Arabidopsis, EDS1 generally interacts with $P A D 4$ and/or $S A G 101$ to defend against pathogen attacks 


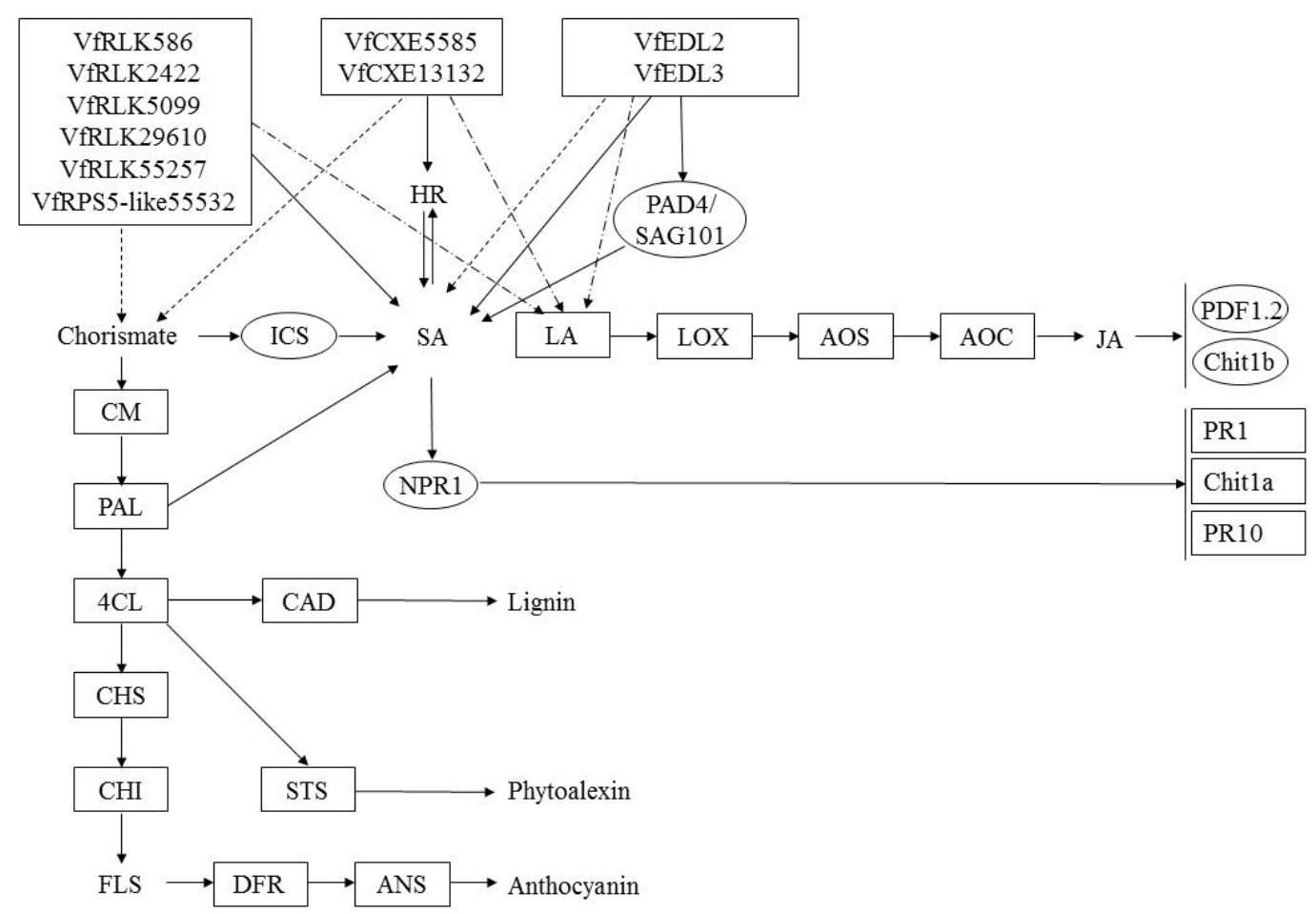

Fig. 10. A diagram of interaction between different $\mathrm{R}$ and $\mathrm{R}$ related genes of Vitis flexuosa and Rhizobium vitis. In this diagram known defense signaling pathways-SA and JA and flavonoid biosynthesis related major genes are used. Upregulated genes are shown by rectangular boxes.

HR: hypersensitive response, PAD4: phytoalexin Deficient4, SAG101: senescence Associated Gene101, ICS: isochorismate synthase, SA: salicylic acid, LA: linoleic acid, $L O X$ : lipoxygenase, AOS: allene oxide synthase, $A O C$ : allene oxide cyclase, JA: jasmonic acid, PDF1.2: plant defensing1.2, Chit1b: chitinase 1b, CM: chorismate mutase, $P A L$ : phenylalanine ammonia-lyase, NPR1: non-expressor of pathogenesis-related genes 1, PR1: pathogenesis related gene 1, Chit1a: chitinase 1a, PR10: pathogenesis related gene 10, 4CL: 4-Coumarate-CoA ligase1, CAD: cinnamyl alcohol dehydrogenase, $C H S$ : chalcone synthase, $C H I$ : chalcone isomerase, STS: stilbene synthase, FLS: flavonol synthase, DFR: dihydroflavonol reductase, ANS: anthocyanidin synthase.

(Feys et al. 2001; Wagner et al. 2013). Additionally, EDS1 can also function independently from PAD4 (Feys et al. 2001). Rietz et al. (2011) has also reported that the dissociated forms of EDS1 and PAD4 are fully competent in signaling receptor triggered localized cell death at infection foci. Consistent with these findings, our results suggested that $V f E D L s$ may function independently in defense signaling pathways.

The results of the present study suggested that $V f R L K$, VfRPS5-like, VfEDL, and VfCXE genes might induce defense responses through the SA-mediated pathway and HR signaling pathway and might result in lignin, phytoalexin and anthocyanin biosynthesis during infection by $R$. vitis. In conclusion, $\mathrm{R}$ and $\mathrm{R}$ related genes in $V$. flexuosa may induce resistant responses to diseases through activation of the SA-mediated pathway rather than JA-mediated pathway genes during the infection of $E$. ampelina, B. cinerea, C. acutatum, and $R$. vitis. In response to $C$. acutatum infection, genes related to both the SA- and JA-mediated pathway are involved in the defense mechanism in $V$. flexuosa, whereas genes involved in the JA-mediated pathway were more active against $E$. necator infection. In the case of genes related to the flavonoid biosynthesis pathway, phytoalexin and lignin biosynthesis were more actively induced than anthocyanin biosynthesis related genes, which indicated that $\mathrm{R}$ related genes may also be closely correlated with phytoalexin and lignin biosynthesis genes in V. flexuosa. Particularly, VfRLK586, 
VfRLK2422, VfRLK5099, VfRLK29610, VfRLK55257, VfRPS5-like4135, VfRPS5-like4832, VfRPS5-like20585, VfRPS5-like55532, VfEDL2, VfEDL3, VfCXE5585, $V f C X E 12827$, and $V f C X E 13132$ genes may be correlated closely with the activation of genes in SA-mediated pathway, lignin, phytoalexin and anthocyanin biosynthesis pathways. VfRLK2422, VfRLK5099, VfRLK29610, VfRPS5-like55532, $V f E D L 3$, and $V f C X E 12827$ may also be activated by defense-related genes in the JA-mediated signaling pathway.

\section{ACKNOWLEDGEMENTS}

This work was supported by the Agricultural $R \& D$ (PJ01163102), Rural Development Administration, Republic of Korea. We thank the Dr. W.K. Kim in National Academy of Agricultural Science, RDA, and Prof. J.S. Cha, Chungbuk National University, Korea for kindly providing the pathogens used in this study.

\section{REFERENCES}

Aarts N, Metz M, Holub E, Staskawicz BJ, Daniels MJ, Parker JE. 1998. Different requirements for EDS1 and $N D R 1$ by disease resistance genes define at least two $R$ gene-mediated signaling pathways in Arabidopsis. Proc. Natl. Acad. Sci. U.S.A. 95: 10306-10311.

Ade J, DeYoung BJ, Golstein C, Innes RW. 2007. Indirect activation of a plant nucleotide binding site-leucine-rich repeat protein by a bacterial protease. Proc. Natl. Acad. Sci. U.S.A. 104: 2531-2536.

Afzal AJ, Wood AJ, Lightfoot DA. 2008. Plant receptor-like serine threonine kinases: roles in signaling and plant defense. Mol. Plant Microbe Interact. 21: 507-517.

Ahn SY, Kim SA, Kim SH, Yun HK. 2012. Differential expression screening of defense related genes in Vitis flexuosa grapevine against Elsinoe ampelina and Rhizobium vitis. Korean J. Intl. Agric. 24: 470-476.

Baudouin E, Charpenteau M, Roby D, Macro Y, Ranjeva R, Ranty B. 1997. Functional expression of a tobacco gene related to the serine hydrolase family: esterase activity towards short-chain dinitrophenyl acylesters. Eur. J.
Biochem. 248: 700-706.

Belhadj A, Saigne C, Telef N, Cluzet S, Bouscaut J, Corio-Costet MF, et al. 2006. Methyl jasmonate induces defense responses in grapevine and triggers protection against Erysiphe necator. J. Agric. Food Chem. 54: 9119-9125.

Bézier A, Lambert B, Baillieul F. 2002. Cloning of a grapevine Botrytis-responsive gene that has homology to the tobacco hypersensitivity-related hsr203J. J. Exp. Bot. 53: 2279-2280.

Chang S, Puryear J, Cairney J. 1993. A simple and efficient method for isolating RNA from pine trees. Plant Mol. Biol. 11: 113-116.

Chen K, Du L, Chen Z. 2003. Sensitization of defense responses and activation of programmed cell death by a pathogen-induced receptor-like protein kinase in Arabidopsis. Plant Mol. Biol. 53: 61-74.

Chen K, Fan B, Du L, Chen Z. 2004. Activation of hypersensitive cell death by pathogen-induced receptor-like protein kinases from Arabidopsis. Plant Mol. Biol. 56: 271-283.

Chong J, Henanff GL, Bertsch C, Walter B. 2008. Identification, expression analysis and characterization of defense and signaling genes in Vitis vinifera. Plant Physiol. Biochem. 46: 469-481.

Dempsey DA, Vlot AC, Wildermuth MC, Klessig DF. 2011. Salicylic acid biosynthesis and metabolism. Arabidopsis Book 9: e156.

Dong X. 2004. NPR1, all things considered. Curr. Opin. Plant Biol. 7: 547-552.

Durrant WE, Dong X. 2004. Systemic acquired resistance. Annu. Rev. Phytopathol. 42: 185-209.

Eibach R, Diehl H, Alleweldt G. 1989. Untersuchungen zur Vererbung von Resistenzeigenschaften bei Reben gegen Oidium tuckeri, Plasmopara viticola und Botrytis cinerea. Vitis 28: 209-228.

Feys BJ, Moisan LJ, Newman MA, Parker JE. 2001. Direct interaction between the Arabidopsis disease resistance signaling proteins, EDS1 and PAD4. EMBO J. 20: 5400-5411.

Feys BJ, Parker JE. 2000. Interplay of signaling pathways in plant disease resistance. Trends Genet. 16: 449-455.

Islam MZ, Ahn SY, Yun HK. 2015a. Analysis of structure and differential expression of Pseudomonas syringae 5-like (RPS5-like) genes in pathogen-infected Vitis 
flexuosa. Turk. J. Biol. 39: 775-789.

Islam MZ, Ahn SY, Yun HK. 2015b. Identification of six transcripts encoding putative receptor-like protein kinase (RLK) and their expression profiles in Vitis flexuosa infected with pathogens. Sci. Hortic. 192: 108-116.

Ko MK, Jeon WB, Kim KS, Lee HH, Seo HH, Kim YS, et al. 2005. A Colletotrichum gloeosporioides-induced esterase gene of nonclimacteric pepper (Capsicum annuum) fruit during ripening plays a role in resistance against fungal infection. Plant Mol. Biol. 58: 529-541.

Kortekamp A, Welter L, Vogt S, Knoll A, Schwander F, Töpfer $\mathrm{R}$, et al. 2008. Identification, isolation and characterization of a CC-NBS-LRR candidate disease resistance gene family in grapevine. Mol. Breed. 22: 421-432.

Kunkel BN, Brooks DM. 2002. Cross talk between signaling pathways in pathogen defense. Curr. Opin. Plant Biol. 5: 325-331.

Lamb C, Dixon RA. 1997. The oxidative burst in plant disease resistance. Ann. Rev. Plant Physiol. 48: 251-275.

Larronde F, Gaudillière JP, Krisa S, Décendit A, Deffieux G, Mérillon JM. 2003. Airborne methyl jasmonate induces stilbene accumulation in leaves and berries of grapevine plants. Am. J. Enol. Viticult. 54: 63-66.

Martin GB. 1999. Functional analysis of plant disease resistance genes and their downstream effectors. Curr. Opin. Plant Biol. 2: 273-279.

Mishina TE, Zeier J. 2007. Pathogen-associated molecular pattern recognition rather than development of tissue necrosis contributes to bacterial induction of systemic acquired resistance in Arabidopsis. Plant J. 50: 500-513.

Pieterse CM, van Loon LC. 1999. Salicylic acid-independent plant defense pathways. Trends Plant Sci. 4: 52-58.

Pieterse CM, Van Loon LC. 2004. NPR1: the spider in the web of induced resistance signaling pathways. Curr. Opin. Plant Biol. 7: 456-464.

Qi D, DeYoung BJ, Innes RW. 2012. Structure-function analysis of the coiled-coil and leucine-rich repeat domains of the RPS5 disease resistance protein. Plant Physiol. 158: 1819-1832.
Reisch BI, Owens CL, Cousins PS. 2012. Grape, p.225-262. In: M.L. Badenes, D.H. Byrne (ed.). Fruit breeding: handbook of plant breeding. Springer, NewYork, NY.

Rietz S, Stamm A, Malonek S, Wagner S, Becker D, MedinaEscobar N, et al. 2011. Different roles of Enhanced Disease Susceptibility1 (EDS1) bound to and dissociated from Phytoalexin Deficient4 (PAD4) in Arabidopsis immunity. New Phytol. 191: 107-119.

Shiu SH, Bleecker AB. 2001. Plant receptor-like kinase gene family: diversity, function, and signaling. Sci. STKE. (113):re22.

Swiderski MR, Innes RW. 2001. The Arabidopsis PBS1 resistance gene encodes a member of a novel protein kinase subfamily. Plant J. 26: 101-112.

Tronchet M, Ranty B, Macro Y, Roby D. 2001. HSR203 antisense suppression in tobacco accelerates development of hypersensitive cell death. Plant J. 27: 115-127.

Tsuda K, Sato M, Glazebrook J, Cohen JD, Katagiri F. 2008. Interplay between MAMP-triggered and SA-mediated defense responses. Plant J. 53: 763-775.

Vlot AC, Dempsey DA, Klessig DF. 2009. Salicylic acid, a multifaceted hormone to combat disease. Ann. Rev. Phytopathol. 47: 177-206.

Wagner S, Stuttmann J, Rietz S, Guerois R, Brunstein E, Bautor J, et al. 2013. Structural basis for signaling by exclusive EDS1 heteromeric complexes with SAG101 or PAD4 in plant innate immunity. Cell Host Microbe 14: 619-630.

Wang Q, Zhang Y, Gao M, Jiao C. Wang X. 2011. Identification and expression analysis of a pathogen-responsive PR-1 gene from Chinese wild Vitis quinquangularis. Afr. J. Biotechnol. 10: 17062-17069.

Xu J, Zhang S. 2015. Mitogen-activated protein kinase cascades in signaling plant growth and development. Trends Plant Sci. 20: 56-64.

Zhou J, Loh YT, Bressan RA, Martin GB. 1995. The tomato gene Ptil encodes a serine/threonine kinase that is phosphorylated by Pto and is involved in the hypersensitive response. Cell 83: 925-935. 